\title{
Minimally invasive, sutureless, adolescent male circumcision with topical anesthetic: a field trial of Unicirc, a single-use surgical instrument
}

\author{
Peter S. Millard ${ }^{1}$, Norman Goldstuck ${ }^{2}$ \\ ${ }^{1}$ Department of Family Medicine, University of New England, Biddeford, Maine, USA; ${ }^{2}$ Department of Obstetrics and Gynaecology, Faculty of \\ Health Sciences, University of Stellenbosch, Cape Town, South Africa \\ Contributions: (I) Conception and design: PS Millard; (II) Administrative support: None; (III) Provision of study materials or patients: None; (IV) \\ Collection and assembly of data: N Goldstuck; (V) Data analysis and interpretation: PS Millard; (VI) Manuscript writing: All authors; (VII) Final \\ approval of manuscript: All authors. \\ Correspondence to: Norman Goldstuck. Department of Obstetrics and Gynaecology, Faculty of Health Sciences, University of Stellenbosch, Cape \\ Town, South Africa. Email: nahumzh@gmail.com.
}

Background: Circumcision has been shown to reduce the rate of HIV transmission in Africa. It is most
cost effective if performed in younger men. Surgical assist devices can increase the efficiency and potentially
reduce the cost of performing circumcisions.

Methods: We used the Unicirc disposable instrument to perform circumcisions in an outpatient primary care clinic. The trial was non-blinded. Circumcisions were performed under topical anaesthetic and the wound was sealed with cyanoacrylate tissue adhesive. The primary outcome was intraoperative duration; secondary outcomes were intraoperative and postoperative pain; adverse events (AEs); time to healing and patient satisfaction; and, cosmetic result.

Results: A total of 82 adolescent boys (aged 10-15 years) were circumcised. The median intraoperative time was 10 minutes and the median blood loss was $1 \mathrm{~mL}$. All wounds were healed by 4 weeks and cosmetic results were excellent. There were no AEs.

Conclusions: Adolescent circumcision with Unicirc under topical anesthetic and wound sealing with cyanoacrylate tissue adhesive is safe, rapid, and heals by primary intention with excellent cosmetic results. It is cost effective and can be used for large scale programs.

Keywords: Circumcision; HIV prevention; minimally invasive; surgical instrument; disposable instrument; topical anaesthetic; tissue adhesive

Submitted Sep 15, 2019. Accepted for publication Nov 29, 2019.

doi: $10.21037 /$ tau.2019.12.16

View this article at: http://dx.doi.org/10.21037/tau.2019.12.16

\section{Introduction}

UNAIDS (Joint United Nations program on HIV/AIDS) "Fast-track" plan to end the global AIDS (Acquired Immune Deficiency Syndrome) epidemic calls for 27 million male circumcisions by 2020 in high prevalence countries of southern and eastern Africa, in addition to the 11.6 million already performed by the end of 2015 (1).

One of the surprising findings of the UNAIDS circumcision program thus far is the unexpectedly lower participation of men, and the higher participation than expected of adolescent boys 10-19 years of age (2). Postpubertal adolescents are likely to be sexually active, with self-reported rates ranging from $9 \%$ in Tanzania to $20.7 \%$ in Zimbabwe (with corresponding HIV risk) (2). Medical circumcision in this age group is similar in technique and complications to that of adult men, although traditional circumcisions have a high complication rate (3).

Circumcision of pre-pubertal boys, on the other hand, 
is technically challenging. In industrialized countries, circumcision of pre-pubertal boys is generally performed by specialists under general anaesthesia, but is performed under local anaesthesia in existing field programs in Africa. The smaller size of the penis presents a challenge for non-specialists without magnifying lenses or cautery instruments, and young boys' anxiety further complicates surgery. As a result, adverse events (AEs) in pre-pubertal boys are far higher than they are among infants or adults (4).

Neonatal circumcision is commonly performed among Jews and Moslems, in West Africa, and in the US, but is not considered a cultural norm in the high HIV prevalence countries of eastern and southern Africa (5). In the US, where over 1 million neonates are circumcised annually, the Gomco ${ }^{\circledR}$ (Alimed, California, USA) clamp is the most commonly used surgical instrument. When used as directed, it has "an impeccable safety record, as the stainless-steel bell protects the glans from the risk of amputation" (6). The disadvantage of the Gomco is that it is a sterilisable instrument that could potentially lead to cross-contamination if not adequately sterilized under field conditions.

The Unicirc surgical instrument functions similarly to the Gomco clamp and has been evaluated in 3 separate studies in adults (7-9). It is a single-use-only metal and plastic disposable surgical instrument. Like the Gomco, Unicirc creates circumferential compression of the base of the foreskin, which fuses the mucosal and skin surfaces, allowing for bloodless excision of the foreskin. Outside of the neonatal period, the fused tissues can easily separate and cause bleeding without further intervention. However, if the fused skin edges are sealed with cyanoacrylate adhesive, there is no open wound and healing occurs by primary intention without use of sutures.

This field trial is the first to evaluate Unicirc circumcision in boys aged 10-15 years. The surgery was conducted in general practice exam rooms, and we evaluated clinical outcomes and AEs.

\section{Methods}

We performed this study according to the guidelines found in the WHO (World Health Organization) Framework for Clinical Evaluation of Devices for Adult Male Circumcision (Framework) (10). Unicirc ${ }^{\circledR}$ (Kapasa Medical, Vienna, Austria) is approved by the South African (SA) Health Products Regulatory Authority and is CE (Conformité Européenne) certified. The SA Medical Association's Ethics
Committee (SAMAREC) approved Unicirc for Phase IV (post-licensing) use (06 March, 2014). The Unicirc disposable devices were supplied by the manufacturer.

We obtained written informed assent from each participant and informed consent from each parent or guardian. The procedures took place between June 2016 and July, 2017. The ClinicalTrials.gov identifier is NCT02593630.

This was a single-center field trial. Healthy uncircumcised boys 10-15 years of age were eligible for the study. Participants were recruited via posters and word of mouth at a primary health care clinic in Mitchell's Plain, Western Cape, South Africa. We excluded volunteers with current illness, bleeding disorder, reaction to local anesthetic, infection, or any penile abnormality potentially complicating circumcision.

We did not perform HIV testing prior to the circumcision and had no information concerning HIV status. We did not inquire about sexual activity.

\section{Surgical procedure}

Two generalist doctors, experienced in surgical and Unicirc circumcision, performed all circumcisions assisted by a single registered nurse in individual consultation rooms in the primary health care clinic.

The primary care doctor applied 3-5 gm of topical Lidocaine and prilocaine (EMLA $\left.{ }^{\mathrm{TM}}\right) 30$ minutes prior to procedure. He determined the size of the instrument needed using a disposable sizing plate. Prior to use, the instruments were gas sterilized in sealed packages. After retracting the foreskin and cleansing the glans and foreskin with povidone iodine, the Unicirc instrument was applied to the glans; the foreskin was then pulled over the transparent bell with the doctor's fingers and adjusted to ensure adequate removal of foreskin. No surgical instruments were used to position the instrument, unless the prepuce required dilation. The Unicirc was then tightened and left in place for 5 minutes; the foreskin was excised with a \#20 surgical scalpel. The instrument was removed and the fused skinmucosal edges were sealed with cyanoacrylate skin adhesive (Exofin $^{\mathrm{TM}}$, Chemence Medical Inc., Alpharetta, Georgia, USA or Indermil ${ }^{\mathrm{TM}}$, Connexicon Medical Ltd., Tallaght, Dublin, Ireland). Operative duration was timed from the beginning of the procedure until the dressing was placed (i.e., including the 5 -minute waiting time). The penis was dressed with adhesive dressing and absorbent gauze. We instructed participants to keep the wound dry, to leave the 
Table 1 Outcome definitions

\begin{tabular}{ll}
\hline Outcome & Definition \\
\hline Pain assessment using Visual Analog Scale [0-10] & Self-reported intraoperative and immediate postoperative pain \\
Blood loss & Estimated by surgeon (mL) \\
Intraoperative duration & From the moment the procedure was started until dressing placed \\
Adverse event & Mild adverse events: no active intervention other than wound pressure for bleeding \\
& Moderate events: medical intervention (sutures, antibiotics) \\
Wound infection & Severe events: transfusion; hospitalization; or resulted in permanent disfigurement \\
Wound disruption & Wound swelling, redness, pain, purulent discharge \\
Wound fully healed & Length of wound disruption (<2 vs. >2 cm) \\
Cosmetic appearance & Wound completely closed. No superficial ulcerations or granulation tissue present \\
& Regular: scar line straight with no irregularity \\
& Irregular: some irregularity to scar line \\
Participant satisfaction (5-point Likert scale) & Scalloped: wavy appearance to scar line \\
\hline
\end{tabular}

gauze in place until it became soiled, and to remove the dressing after 3 weeks if it was still in place.

We observed participants for 30 minutes after the procedure, and gave their parent/guardian written postoperative instructions with cellular telephone contact information of the doctor.

The primary outcome measured was intraoperative duration and the secondary outcome measures included intraoperative and postoperative pain; AEs (intraoperative and postoperative); wound disruption, healing at 4 weeks; patient satisfaction; and, cosmetic result. The WHO Framework served as the guideline for evaluating AEs. Mild AEs require little or no intervention (e.g., slight bleeding), moderate AEs require active treatment (e.g., antibiotics or suturing), and severe AEs require transfusion or hospitalization, or result in permanent damage. Key AEs evaluated were anaesthetic complications, bleeding, haematoma, infection, problems with urination, subsequent procedures needed to correct complications, or poor cosmetic results.

The sample size was based on the Framework: "Studies involving about 100 (range, 50 to 300) are suggested as a compromise between assessing safety, documenting the presumed advantages of the new method, and ensuring rapid progress through the different stages of clinical assessment. For devices which are aids to surgery and do not stay on the penis... study sizes at the lower end 25-100 range may be sufficient."

All boys were followed up at 7 days and 4 weeks. If the wound was not completely healed by 4 weeks, we planned to conduct a 6-week follow-up visit.

\section{Outcomes}

Outcome definitions are shown in Table 1. The surgeons themselves assessed wound-healing outcomes.

We used a 10 -point visual analog scale for the intraoperative and postoperative pain survey, which was administered prior to leaving the clinic. Satisfaction was assessed at the final visit using a 5-point Likert scale.

\section{Data analysis}

We collected data from participants on socio-demographics and reason for circumcision. We analyzed data with Epi Info, version 7 (Atlanta, USA). For continuous variables, we used nonparametric statistics. For categorical variables, we used the chi-square or Fisher's exact test. All $\mathrm{P}$ values were two-sided.

\section{Results}

We recruited participants in 2016 and 2017. Follow-up was completed in July, 2017. A total of 87 boys and their parent/ guardian were interviewed and $82(94.3 \%)$ participated in the study. SN performed 63 circumcisions, and ZP performed 19 circumcisions. 
Table 2 Baseline characteristics

\begin{tabular}{lc}
\hline Baseline characteristic & Unicirc (N=82) \\
\hline Age (years), $\mathrm{n}(\%)$ & $9(11.0)$ \\
$10-11$ & $52(63.4)$ \\
$12-13$ & $21(25.6)$ \\
$14-15$ & 12 \\
Median age (years) & \\
Religion, $\mathrm{n}$ (\%) & $58(70.7)$ \\
Christian & $24(29.3)$ \\
Muslim & \\
Reason for circumcision, $\mathrm{n}(\%)$ & $49(59.8)$ \\
Hygiene & $24(29.3)$ \\
Reduce HIV infection & $9(11.0)$ \\
Social/religious &
\end{tabular}

Table 3 Operative outcomes

\begin{tabular}{lc}
\hline Operative outcome & Unicirc \\
\hline Operative duration in min, median (range) & $10[8-14]$ \\
Estimated blood loss (mL), median (range) & $1[1-2]$ \\
Diameter of Unicirc bell (cm), n (\%) & \\
1.4 & $2(2.4)$ \\
1.6 & $18(21.4)$ \\
2.0 & $3(3.5)$ \\
2.6 & $17(20.2)$ \\
2.9 & $12(14.2)$ \\
3.2 & $18(21.4)$ \\
Intraoperative pain (10-point scale), & $1[1-4]^{\dagger}$ \\
median (range) & $3[0-6]$ \\
Postoperative pain (10-point scale), \\
median (range)
\end{tabular}

${ }^{\dagger}$, one boy cried and gave the pain a rating of $4 / 10$.

The baseline characteristics of the participants are shown in Table 2. Most boys gave improved hygiene as their motivation for circumcision; one-third were motivated by a potential reduction in $\mathrm{HIV}$ infection.

Table 3 shows operative outcomes. Intraoperative and postoperative pain was minimal. Intraoperative duration was 10 minutes (including the 5 minutes when the instrument
Table 4 Adverse events

\begin{tabular}{lc}
\hline Adverse event & Unicirc \\
\hline Intraoperative suturing, $\mathrm{n}$ & 0 \\
Serious postoperative complication, $\mathrm{n}$ & 0 \\
Postoperative bleeding, $\mathrm{n}$ & \\
Mild (dressing only) & 1 \\
Moderate (sutured) & 0 \\
Haematoma, $\mathrm{n}$ & 0 \\
Postoperative infection (requiring antibiotic), $\mathrm{n}$ & 0 \\
\hline
\end{tabular}

Table 5 Postoperative outcomes

\begin{tabular}{lc}
\hline Postoperative outcome & Unicirc \\
\hline Wound disruption, $\mathrm{n}(\%)$ & $2(2.4)$ \\
$<2 \mathrm{~cm}$ length & 0 \\
$>2 \mathrm{~cm}$ length & $82[100]$ \\
Wound fully healed at 4 weeks, $\mathrm{n}(\%)$ & \\
Satisfaction, $\mathrm{n}(\%)$ & $82[100]$ \\
Very satisfied & 0 \\
Satisfied & 0 \\
Not satisfied & \\
Cosmetic results, $\mathrm{n}(\%)$ & $82[100]$ \\
Regular & 0 \\
Irregular & 0 \\
Scalloped & \\
\hline
\end{tabular}

was in place) and blood loss was minimal. There were no differences of operative duration time and surgical outcome between early-pubertal and late-pubertal boys. Adverse events are shown in Table 4.

Healing, participant satisfaction, and cosmetic results are shown in Table 5. No wound disruptions required intervention, and all healed uneventfully. All were fully healed at 4 weeks. Satisfaction was high. The cosmetic result was excellent; a regular scar line was found in all boys.

\section{Discussion}

One of the key goals of the UNAIDS Fast-track plan to end AIDS is the successful completion of 25 million medical male circumcisions by 2020 , in addition to the 11.6 million 
performed as of the end of 2015. Demand for circumcision among adult men has lagged, while demand for circumcision among adolescents has exceeded expectations. In most of the 14 high prevalence countries, over $40 \%$ of circumcisions performed in 2015 were in boys less than 15 years of age, reaching $72 \%$ in Swaziland (2). The relatively low demand in men, and increased demand in young adolescents, may be due to the cultural tradition of circumcision, which sees it as a rite of passage into manhood, and not culturally appropriate for adult men (2). Adolescents generally do not work or have the same financial pressures as men, giving them more flexibility. Given that the average age of sexual debut in the high prevalence regions is approximately 18 years of age, circumcision prior to the onset of sexual activity is advantageous because it obviates concerns about transmission of infection during wound healing. Over the long term, circumcising younger boys delivers the greatest reduction in HIV incidence compared with older age groups (1).

Until now, almost all circumcisions in the 14 high prevalence countries were done with the open surgical method under local anaesthesia, which is technically challenging and has a high complication rate. This study showed that, in boys aged 10-15, the Unicirc method was rapid to perform, caused minimal discomfort, and healed promptly with excellent cosmetic results and no adverse events.

This study involved 2 overlapping populations of boys: early vs. late pubertal. Penile growth is a late pubertal phenomenon, which results in a rapid doubling of penile length. We defined early pubertal boys as having a glans diameter $<2.6 \mathrm{~cm}$ and late pubertal boys as having a glans diameter at least $2.6 \mathrm{~cm}$. By these criteria, $44 \%$ of our participants were pre-pubertal and $64 \%$ were post-pubertal.

The generalist doctors in this study were experienced in both surgical and Unicirc circumcision among men, but had not previously used Unicirc in boys. Operative duration was similar to prior studies. Because the Unicirc operative duration includes 5 minutes of waiting with the Unicirc in situ, the doctors were able to perform multiple procedures sequentially, thus further reducing the time needed (11). Unicirc generally requires no surgical instruments other than a scalpel, no injectable anesthetic, and no sutures. It is completed at one point in time and, unlike plastic ring devices, requires no follow-up visit for device removal.

Follow-up at 4 weeks was complete, and there were no significant adverse events. One boy was kicked in the groin at school 18 days following the circumcision and had mild bleeding which did not require treatment. Given the fact that complications are higher using the surgical method during this age range than at any other time, the absence of complications implies that Unicirc is superior to surgical circumcision in this age group, as previously shown in adults (7-9). Because of the small risk of postoperative bleeding, we recommend that sites which perform Unicirc be capable of suturing. There were two minor wound separations not requiring treatment.

No boys had pathological phimosis, although we have shown in adults that Unicirc works well with concomitant phimosis. Several boys had tight prepuces which required dilation (with a hemostat) prior to placing the Unicirc.

Cyanoacrylate tissue adhesive is superior to sutures in paediatric (12-19) and adult (8,9,20-25) circumcision. We used both high viscosities, quick-setting 2-octyl cyanoacrylate (Exofin ${ }^{\mathrm{TM}}$ ) and a lower viscosity product (Indermil ${ }^{\mathrm{TM}}$ ), each in approximately half the procedures. Both performed similarly, but we found the high viscosity product easier to apply with less running.

This study was limited by the fact that it was a single-arm field trail and not a randomized controlled trial. Wound healing was assessed by the doctors themselves and there was no independent assessment.

Two plastic ring devices have been approved by WHO, but one of those (Prepex) was recently removed from the market. With the remaining approved device (Shang Ring), healing is by secondary intention, leading to delayed healing and potentially increased HIV transmission prior to wound closure. The device is promoted because it is rapidly applied, but the need for a follow-up removable session with specialized instruments obviates any time advantages.

\section{Conclusions}

Unicirc circumcision under topical anaesthetic and wound sealing with cyanoacrylate tissue adhesive in boys 10 to 15 years of age is safe, rapid, heals by primary intention, and has excellent cosmetic results.

\section{Acknowledgments}

We thank Dr. Senzo Ntshalintshali and Dr. Zainul Parker for performing the circumcisions. Drs. Cyril and Elizabeth Parker and the rest of the staff at Simunye Primary Health Care provided support for the study. Simone Harris performed the data entry.

Funding: This study was supported by Simunye Primary Health Care. 


\section{Footnote}

Conflicts of Interest: All authors have completed the ICMJE uniform disclosure form (available at http://dx.doi. org/10.21037/tau.2019.12.16). The authors have no conflicts of interest to declare.

Ethical Statement: The authors are accountable for all aspects of the work in ensuring that questions related to the accuracy or integrity of any part of the work are appropriately investigated and resolved. The SA Medical Association's Ethics Committee (SAMAREC) approved Unicirc for Phase IV (post-licensing) use (06 March, 2014). We obtained written informed assent from each participant and informed consent from each parent or guardian.

Open Access Statement: This is an Open Access article distributed in accordance with the Creative Commons Attribution-NonCommercial-NoDerivs 4.0 International License (CC BY-NC-ND 4.0), which permits the noncommercial replication and distribution of the article with the strict proviso that no changes or edits are made and the original work is properly cited (including links to both the formal publication through the relevant DOI and the license). See: https://creativecommons.org/licenses/by-nc$\mathrm{nd} / 4.0 \%$.

\section{References}

1. Fast Track: Ending the Global AIDS Epidemic by 2030 . Geneva: World Health Organization, 2015.

2. Njeuhmeli E, Hatzold K, Gold E, et al. Lessons learned from scale-up of voluntary medical male circumcision focusing on adolescents: benefits, challenges, and potential opportunities for linkages with adolescent HIV, sexual, and reproductive health services. J Acquir Immune Defic Syndr 2014;66 Suppl 2:S193-9.

3. Wilcken A, Keil T, Dick B. Traditional male circumcision in eastern and southern Africa: a systematic review of prevalence and complications. Bull World Health Organ 2010;88:907-14.

4. Weiss HA, Larke N, Halperin D, et al. Complications of circumcision in male neonates, infants and children: a systematic review. BMC Urol 2010;10:2.

5. Westercamp N, Bailey RC. Acceptability of male circumcision for prevention of HIV/AIDS in sub-Saharan Africa: a review. AIDS Behav 2007;11:341-55.

6. Manual for early infant male circumcision under local anaesthesia. Geneva: World Health Organization, 2010.

7. Shenje J, Millard PS. Sutureless Adult Voluntary Male Circumcision with Topical Anaesthetic: A Randomized Field Trial of Unicirc, a Single-Use Surgical Instrument. PLoS One 2016;11:e0157065.

8. Millard PS, Goldstuck ND. No-needle, single-visit adult male circumcision with Unicirc: a multi-centre field trial. PLoS One 2015;10:e0121686.

9. Millard PS. Rapid, Minimally-invasive Voluntary Adult Male Circumcision: a Quasi-experimental Study (NCT01998360). Available online: http://clinicaltrials.gov/ ct2/show/NCT01998360? term=unicirc\&rank=2

10. Framework for Clinical Evaluation of Devices for Adult Male Circumcision. Geneva: World Health Organization, 2011.

11. Considerations for implementing models for optimizing the volume and efficiency of male circumcision services. Geneva: World Health Organization; 2010. Available online: http://www.malecircumcision.org/programs/ documents/mc_MOVE_2010_web.pdf

12. Elemen L, Seyidov TH, Tugay M. The advantages of cyanoacrylate wound closure in circumcision. Pediatr Surg Int 2011;27:879-83.

13. Elmore JM, Smith EA, Kirsch AJ. Sutureless circumcision using 2-octyl cyanoacrylate (Dermabond): appraisal after 18-month experience. Urology 2007;70:803-6.

14. Kaye JD, Kalisvaart JF, Cuda SP, et al. Sutureless and scalpel-free circumcision--more rapid, less expensive and better? J Urol 2010;184:1758-62.

15. Kelly BD, Lundon DJ, Timlin ME, et al. Paediatric sutureless circumcision--an alternative to the standard technique. Pediatr Surg Int 2012;28:305-8.

16. Lane V, Vajda P, Subramaniam R. Paediatric sutureless circumcision: a systematic literature review. Pediatr Surg Int 2010;26:141-4.

17. Van Haute C, Tailly T, Klockaerts K, et al. Sutureless circumcision using 2-Octyl cyanoacrylate results in more rapid and less painful procedures with excellent cosmetic satisfaction. J Pediatr Urol 2015;11:147.e1-5.

18. Gorgulu T, Olgun A, Torun M, et al. A fast, easy circumcision procedure combining a $\mathrm{CO} 2$ laser and cyanoacrylate adhesive: a non-randomized comparative trial. Int Braz J Urol 2016;42:113-7.

19. Voznesensky M, Mutter C, Hayn M, et al. Pediatric sutureless circumcision: an effective and cost efficient alternative. Can J Urol 2015;22:7995-9.

20. Millard PS, Wilson HR, Goldstuck ND, et al. Rapid, minimally invasive adult voluntary male circumcision: A 
randomised trial of Unicirc, a novel disposable device. S Afr Med J 2013;104:52-7.

21. Millard PS, Wilson HR, Veldkamp PJ, et al. Rapid, minimally invasive adult voluntary male circumcision: A randomised trial. S Afr Med J 2013;103:736-42.

22. O'Sullivan J, Cahill K, Shabbir J, et al. Circumcision: Gomco Clamp Method, the possible benefit of tissue glue over sutures. Research Bulletin, Irish Nat Inst Health Sci $2002 ; 1: 14$.

Cite this article as: Millard PS, Goldstuck N. Minimally invasive, sutureless, adolescent male circumcision with topical anesthetic: a field trial of Unicirc, a single-use surgical instrument. Transl Androl Urol 2020;9(2):516-522. doi: 10.21037/ tau.2019.12.16
23. O'Sullivan J, Shabbir J, Masokwane P, et al. The benefits of histoacryl tissue glue over sutures for wound approximation after circumcision with Gomco clamp. Irish J Med Sci 2002;171:57-8.

24. Sharma P. Sutureless circumcision: Wound closure after circumcision with cynoacrylate glue - A preliminary Indian study. Indian J Surg 2004;66:276-8.

25. Tiwari P, Tiwari A, Kumar S, et al. Sutureless circumcision - An Indian experience. Indian J Urol 2011;27:475-8. 